\section{Spectrum of pathogenic alterations identified after two decades of $H B B$ gene sequencing for molecular diagnosis of beta-thalassaemias and haemoglobinopathies}

Sir,

Inherited haemoglobin disorders, including reduced globin expression ( $\beta$-thalassaemia) and structural haemoglobin variants (haemoglobinopathies) are common genetic blood disorders worldwide. ${ }^{1}$ Screening is performed using a full blood count, haemoglobin analysis of haemoglobin A2 (HbA2) and haemoglobin $\mathrm{F}(\mathrm{HbF})$ by capillary electrophoresis or high performance liquid chromatography, and gel electrophoresis. ${ }^{2}$ Microcytic anaemia with an elevated level of $\mathrm{HbA} 2$ or $\mathrm{HbF}$ suggests $\beta$-thalassaemia or a haemoglobinopathy, which are caused by pathogenic alternations in the beta globin $(H B B)$ gene. Located on the short arm of chromosome 11 (11p15.5), the $H B B$ gene consists of three exons encoding 147 amino acids. The majority (95\%) of $\beta$-thalassaemias and haemoglobinopathies are due to small nucleotide substitutions, deletions or insertions, and only a minority of $\beta$-thalassaemias are due to large gene deletions. ${ }^{2}$ Currently there are at least $800 \mathrm{HBB}$ pathogenic alterations reported in curated databases including $\mathrm{HbVar}^{3}{ }^{3}$ IthaGenes $^{1}$ and HGMD. ${ }^{4}$ Different ethnic groups and populations from different geographical locations have distinct mutational spectra of $H B B$ variants. 5,6 Many molecular laboratories adopt targeted mutational analysis using reverse dot blot or primer specific amplification assays to detect commonly occurring variants in the populations from which their patients originate. While being cost effective and time efficient, targeted testing may miss rare and novel variants. Mutation scanning and sequence analysis have an analytical sensitivity of $99 \%$ for mutation detection. ${ }^{7}$ Capillary sequencing is the best current method for interrogating all possible small genetic variants in the $H B B$ gene.

Located in Singapore, a city with a multi-ethnic South East Asian population with a substantial proportion of international immigrants, our laboratory has been using capillary sequencing to genotype the $H B B$ gene (NM_000518.5) in patients with clinical suspicion of $\beta$-thalassaemia and haemoglobinopathies since 1998. Genomic DNA is extracted from whole blood and polymerase chain reaction (PCR) is performed using a set of primers flanking the nucleotides c.177 and c. $* 459$ to generate a PCR product of 2060 nucleotides, which is then run on gel electrophoresis. Subsequently, the purified PCR product is sequenced with ABI BigDye Terminator v1.1 and v3.1 chemistries (Thermo Fisher Scientific, Australia) using multiple forward and reverse sequencing primers followed by capillary electrophoresis on ABI Genetic Analysers 310 and 3130XL (Thermo Fisher Scientific). All primers used have been checked for specificity and possible mismatches using PrimerBLAST ${ }^{8}$ and SNPCheck V3 (https://genetools.org/SNPCheck/credits. $\mathrm{htm}$ ), respectively. Sequence analysis is performed for the entire coding region and clinically relevant non-coding regions of the $H B B$ gene including the proximal promoter, $5^{\prime}$ untranslated region (UTR), partial introns 1 and 2, 3' UTR and the polyadenylation signal site. Notably, the 619-bp deletion (HBB:c.316-149_*342delinsAAGTAGA), which is known to be frequently detected in patients of Indian ethnicity, has been identified by detecting the $1.4 \mathrm{~kb}$ PCR product on gel electrophoresis with the deletional breakpoint ascertained by capillary sequencing.

Of a total of 825 individual patients who have had their $H B B$ gene sequenced since 1998, 25 (3.0\%) had pathogenic variants in homozygous state and $39(4.7 \%)$ had two different heterozygous pathogenic variants. Another 560 patients $(67.9 \%)$ were carriers of one pathogenic variant. Our capillary sequencing assay did not identify any pathogenic variants in 201 patients $(24.4 \%)$. This could be due to the patients having other rare large deletional variants causing their clinical symptoms, or more likely it is due to a low pre-test probability as the patients' blood samples were sent for genetic testing as part of asymptomatic prenatal screening for thalassaemias.

A total 48 different pathogenic alterations were found in our patients (Fig. 1). The top ten most frequently identified pathogenic alterations were: c.79G $>\mathrm{A}$ ( $\mathrm{Hb} \mathrm{E} ; 20.6 \%)$, c.126_129delCTTT (Codons 41/42 -TTCT; 15.8\%), c.316$197 \mathrm{C}>\mathrm{T}$ (IVS-II-654 C $>\mathrm{T} ; 14.4 \%), \mathrm{c} .92+5 \mathrm{G}>\mathrm{C}$ (IVS-I-5 $\mathrm{G}>\mathrm{A} ; 10.5 \%)$, с. $-78 \mathrm{~A}>\mathrm{G}(-28 \mathrm{~A}>\mathrm{G} ; 4.8 \%), \quad$ c. $52 \mathrm{~A}>\mathrm{T}$ (Codon $17 \mathrm{~A}>\mathrm{T} ; 3.9 \%$ ), c.316-238C $>\mathrm{T}$ (IVS-II-613 C $>\mathrm{T}$; $3.9 \%)$, c. $20 \mathrm{~A}>\mathrm{T}(\mathrm{Hb} \mathrm{S} ; 3.6 \%)$, c. $92+1 \mathrm{G}>\mathrm{T}(\mathrm{IVS}-\mathrm{I}-1 \mathrm{G}>\mathrm{T}$; $3.2 \%$ ) and c.316-149_*342delinsAAGTAGA (619 bp deletion; $3.1 \%$ ). Together, these contributed to $83.9 \%$ of all the pathogenic alterations identified. These results are consistent with a previous study by $\mathrm{Ng}$ et al. which found that the c.126_129delCTTT, c.316-197C $>$ T, c. $92+5 \mathrm{G}>$ C, c.$78 \mathrm{~A}>\mathrm{G}$ and $\mathrm{c} .52 \mathrm{~A}>\mathrm{T}$ variants were among the most common mutations in $\beta$-thalassaemia patients in the Singapore population. ${ }^{9}$ A further 38 pathogenic variants accounted for the remaining $16.2 \%$ of $H B B$ pathogenic alterations identified in our patients (Fig. 1). The broad mutational spectrum of $H B B$ pathogenic alterations identified may be due to an increase in immigrants and/or international patients, as other studies have shown that migration can broaden the range of haemoglobinopathy mutations. ${ }^{10}$ If the $\beta$-Globin StripAssay SEA (ViennaLab Diagnostics GmbH, Austria) targeted panel had been used (which claims to detect $>90 \%$ of $\beta$-globin defects found in South East Asia), only 18 of the 48 identified pathogenic alterations would have been detected (Fig. 1), translating into an expected analytical sensitivity of $80.3 \%$ in comparison to our current sequencing assay. This demonstrates the value of the capillary sequencing-based approach for molecular diagnosis of $\beta$-thalassaemias and haemoglobinopathies by detecting many rare pathogenic variants in addition to those common pathogenic variants.

However, with the whole gene analysis approach by capillary sequencing, we are occasionally challenged with interpretation of the pathogenicity of rare and novel variants. We detected six heterozygous variants of uncertain significance (VUS) in the $H B B$ gene (Table 1). They are located in the $5^{\prime}$ UTR or intron 2 of the gene, which are known to harbour clinically significant variants. These variants are not present in the HbVar, IthaGenes and HGMD databases. 


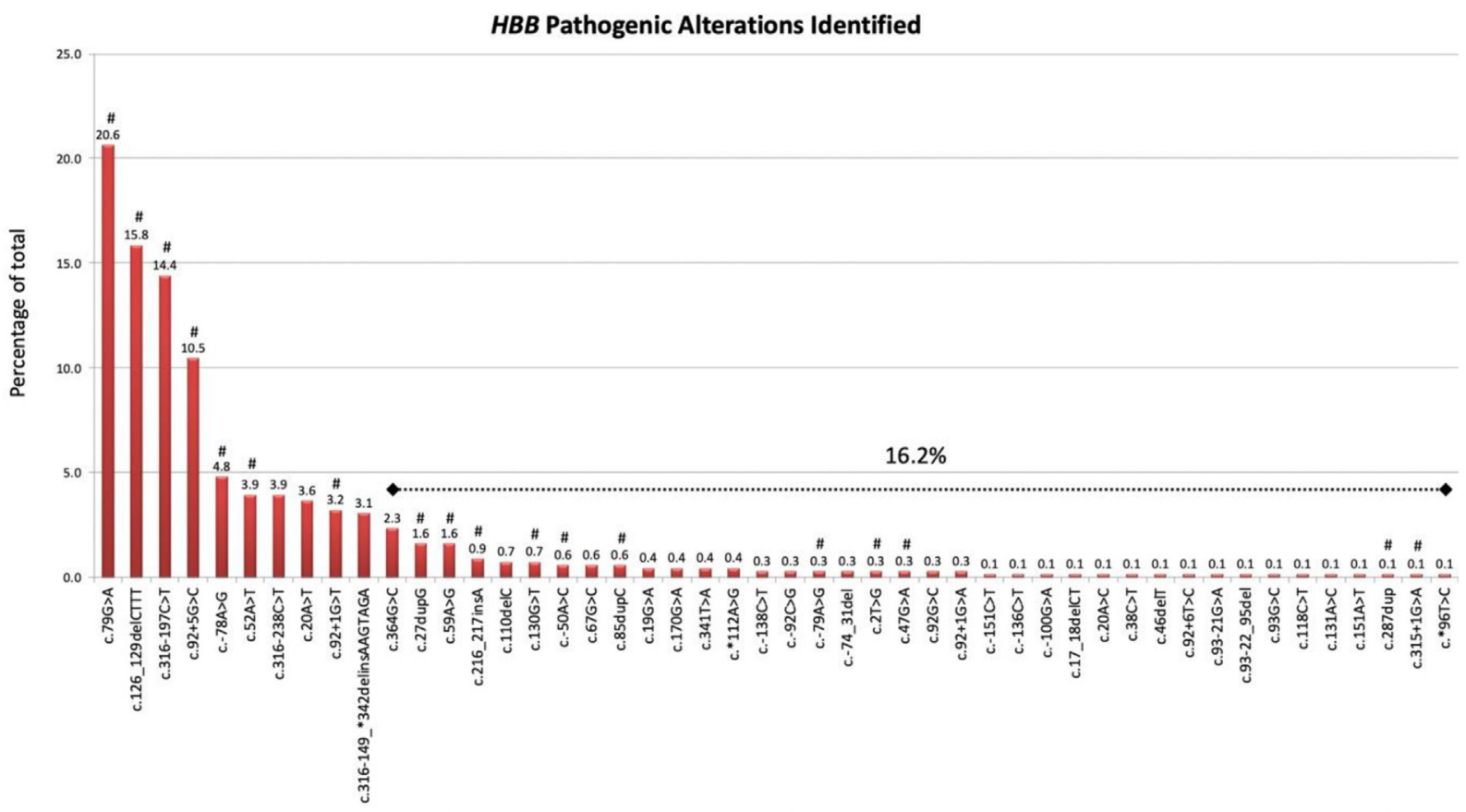

Fig. 1 Mutational spectrum of $H B B$ pathogenic variants identified in 624 patients. Variants are shown in decreasing order of frequency as a percentage of the total number of variants identified. A total 48 different pathogenic alterations were found. The top ten most frequently identified pathogenic alternations are c.79G $>A$, c.126_129delCTTT, c.316-197C $>$ T, c.92+5G >C, c.-78A $>$ G, c.52A $>$ T, c.316-238C $>$ T, c.20A $>$ T, c.92+1G $>$ T and c.316-149_*342delinsAAGTAGA. The other 38 minority pathogenic variants accounted for $16.2 \%$ of all pathogenic alterations. " Variants detectable by the $\beta$-Globin StripAssay SEA (ViennaLab Diagnostics GmbH) targeted panel.

Table 1 Variants of unknown significance (VUS) identified in the $H B B$ gene in our laboratory

\begin{tabular}{|c|c|c|c|c|c|c|c|c|c|}
\hline HGVS name & Legacy name & Location & dbSNP ID & $\begin{array}{l}\text { ClinVar } \\
\text { accession ID; } \\
\text { interpretation }\end{array}$ & $\begin{array}{l}\text { gnomAD } \\
\text { v3.0 allele } \\
\text { frequency }\end{array}$ & $\begin{array}{c}\mathrm{Hb} \\
(11.4-16.6 \mathrm{~g} / \mathrm{dL})\end{array}$ & $\begin{array}{c}\text { MCV } \\
(83.0-95.5 \mathrm{fL})\end{array}$ & $\begin{array}{c}\mathrm{MCH} \\
(26.5-31.5 \mathrm{pg})\end{array}$ & $\begin{array}{c}\mathrm{HbA} 2 \\
(\%)\end{array}$ \\
\hline c. $-84 \mathrm{G}>\mathrm{A}$ & $-34 \mathrm{G}>\mathrm{A}$ & $5^{\prime} \mathrm{UTR}$ & NA & NA & NA & 12.9 & 76.4 & 23.4 & 2.4 \\
\hline c. $-51 \mathrm{~T}>\mathrm{C}^{\mathrm{a}}$ & $-1 \mathrm{~T}>\mathrm{C}$ & $5^{\prime} \mathrm{UTR}$ & rs386134236 & $\begin{array}{l}\text { VCV000036293.3; } \\
\text { VUS }\end{array}$ & $4.05 \mathrm{e}-4$ & 11.8 & 62.3 & 20.5 & 2.6 \\
\hline c. $-11 \mathrm{~A}>\mathrm{G}$ & $+40 \mathrm{~A}>\mathrm{G}$ & $5^{\prime} \mathrm{UTR}$ & rs1447873672 & NA & NA & 11.7 & 72.3 & 23.4 & ND \\
\hline c. $316-233 \mathrm{~T}>\mathrm{C}$ & IVS-II-618 T>C & Intron 2 & NA & NA & NA & ND & ND & ND & ND \\
\hline $\begin{array}{l}\mathrm{c} .316- \\
100 \mathrm{~T}>\mathrm{A}\end{array}$ & IVS-II-751 T >A & Intron 2 & NA & NA & NA & 12.6 & 89.8 & 30.6 & 2.5 \\
\hline c. $316-45 \mathrm{G}>\mathrm{C}$ & IVS-II-806 G $>C$ & Intron 2 & rs140033163 & $\begin{array}{l}\text { VCV000495995.5; } \\
\text { conflicting } \\
\text { interpretations of } \\
\text { pathogenicity }\end{array}$ & $2.44 \mathrm{e}-4$ & $\begin{array}{l}10.3 \\
14.6\end{array}$ & $\begin{array}{l}64.3 \\
82.6\end{array}$ & $\begin{array}{l}20.7 \\
27.4\end{array}$ & $\begin{array}{l}2.7 \\
2.6\end{array}$ \\
\hline
\end{tabular}

$\mathrm{Hb}$, haemoglobin; $\mathrm{MCH}$, mean corpuscular haemoglobin; MCV, mean corpuscular volume; NA, not present in the database; ND, no data available.

a The patient with the c.-51T $>\mathrm{C}$ variant was also heterozygous for $H B B$ :c.-136C $>\mathrm{T}$ pathogenic variant as well as the $\alpha$-globin South East Asian deletion $(--\mathrm{SEA} / \alpha \alpha)$.

Three variants, c.-84G $>$ A, c.316-233T $>C$ and c.316$100 \mathrm{~T}>\mathrm{A}$, are not present in dbSNP, ClinVar and population databases such as gnomAD, and appear to be novel variants. The MCV, MCH and $\mathrm{HbA} 2$ of the individuals with the c.$84 \mathrm{G}>\mathrm{A}$ and c.316-100T $>\mathrm{A}$ variants do not appear very abnormal; unfortunately no data from family members was available to help interpret the pathogenicity of the variants. The individual with the c.-51T $>\mathrm{C}$ variant also was a carrier of the South East Asian deletion (-SEA/ $\alpha \alpha)$ and had a $H B B:$ : $-136 \mathrm{C}>\mathrm{T}$ pathogenic variant. Capillary sequencing was unable to discriminate whether the c.-51T $>\mathrm{C}$ and c.$136 \mathrm{C}>\mathrm{T}$ variants are in cis on the same allele or in trans on two different alleles of the $H B B$ gene in this individual. The c. $316-45 \mathrm{G}>\mathrm{C}$ variant was detected in seven individuals. This variant has been described as benign, likely benign and of uncertain significance in ClinVar. The two individuals with this variant with available haemoglobin analysis did not have elevated $\mathrm{HbA} 2$. Thus, this variant is likely benign.

The full gene sequencing approach improves the molecular diagnosis of $\beta$-thalassaemia and haemoglobinopathies by increasing the total number of pathogenic variants identified. Although the occurrence of each individual rare pathogenic variant is low, together they make up to more than $10 \%$ of the total identifiable variants in our test population, which could have been potentially missed if a targeted approach detecting only ethnic or population specific variants was applied. The 
identification of all pathogenic variants in the patients is important for genetic counselling for couples regarding the risk to their offspring.

Conflicts of interest and sources of funding: The authors state that there are no conflicts of interest to disclose.

\section{Kok-Siong Poon, Karen Mei-Ling Tan}

Department of Laboratory Medicine, National University Hospital, Singapore

Contact Kok-Siong Poon.

E-mail: kok_siong_poon@nuhs.edu.sg

1. Kountouris P, Lederer CW, Fanis P, Feleki X, Old J, Kleanthous M IthaGenes: an interactive database for haemoglobin variations and epidemiology. PLoS One 2014; 9: e103020.

2. Sabath DE. Molecular diagnosis of thalassemias and hemoglobinopathies: an ACLPS critical review. Am J Clin Pathol 2017; 148: 6-15.

3. Giardine B, Borg J, Viennas E, et al. Updates of the HbVar database of human hemoglobin variants and thalassemia mutations. Nucleic Acids Res 2014; 42: D1063-9.

4. Stenson PD, Ball EV, Mort M, et al. Human gene mutation database (HGMD): 2003 update. Hum Mutat 2003; 21: 577-81.

5. Fucharoen S, Winichagoon P. Hemoglobinopathies in Southeast Asia: molecular biology and clinical medicine. Hemoglobin 1997; 21: 299-319.

6. Kumar R, Sagar C, Sharma D, Kishor P. $\beta$-globin genes: mutation hotspots in the global thalassemia belt. Hemoglobin 2015; 39: 1-8.

7. Cao A, Galanello R. Beta-thalassemia. Genet Med 2010; 12: 61-76.

8. Ye J, Coulouris G, Zaretskaya I, Cutcutache I, Rozen S, Madden TL. Primer-BLAST: a tool to design target-specific primers for polymerase chain reaction. BMC Bioinform 2012; 13: 134

9. Ng IS, Ong JB, Tan CL, Law HY. Beta-thalassemia mutations in Singapore-a strategy for prenatal diagnosis. Hum Genet 1994; 94: 385-8.

10. Henderson S, Timbs A, McCarthy J, et al. Incidence of haemoglobinopathies in various populations - the impact of immigration. Clin Biochem 2009; 42: 1745-56.

DOI: https://doi.org/10.1016/j.pathol.2020.08.022

\section{Variability in D-dimer reporting revisited}

Sir,

D-dimers represent a breakdown product of fibrin formation, and D-dimer testing is a common laboratory procedure in haemostasis laboratories. ${ }^{1} \mathrm{D}$-dimer testing may be requested in patients as an investigative tool for assessment of venous thromboembolism (VTE), such as deep vein thrombosis (DVT) or pulmonary embolism (PE), typically combined with a pre-test probability score (e.g., Well's score), or else for assessment and potential monitoring of disseminated intravascular coagulation (DIC). ${ }^{1}$ Of particular relevance to the current report, is that D-dimer testing has found particular utility as a potential prognostic marker for disease severity in coronavirus disease 2019 (COVID-19), which characterises a pandemic produced by severe acute respiratory syndrome virus coronavirus 2 (SARS-CoV-2). At time of writing, COVID-19 comprised over 17 million confirmed cases, causing nearly 700,000 deaths. ${ }^{2}$ The disease expresses various pathophysiological derangements, including (micro) thrombosis, ${ }^{3-5}$ which in turn is associated with various derangements of haemostasis parameters, in particular including D-dimer. ${ }^{6}$ As noted, D-dimer also potentially serves as a prognostic marker for severe disease and/or mortality. ${ }^{7}$ Thus, it is anticipated that D-dimer testing will increase substantially as clinicians assess and treat increasing numbers of COVID-19 patients.

Of additional relevance to this correspondence is wide under-recognition of the substantial variation in D-dimer reporting units, ${ }^{8}$ and thus also the likelihood of misreporting D-dimer data because of poor or incomplete information. Although at least 28 potential theoretical combinations of D-dimer units can be identified, ${ }^{8}$ a summary of the eight most common was recently identified, ${ }^{9}$ including recognition that different manufacturers of D-dimer reagents report in several different preferential units. Two layers of possible misreporting exist. The first reflects using either D-dimer units (DDU) or fibrinogen equivalent units (FEU), the latter being almost $2 \times$ those of DDU. The second is the actual measuring units used: these may be in $\mathrm{ng}, \mu \mathrm{g}, \mathrm{mg}$, or $\mathrm{g}$, per $\mathrm{mL}, \mathrm{L}$ and potentially even $\mu \mathrm{L}$. This secondary layer creates the possibility of some 1000-fold difference in reporting values, which combined with the first layer leads to the possibility of a 2000-fold error in reporting values.?

Given the recent assessment showing several errors in Ddimer reporting in the COVID-19 literature, ${ }^{9}$ and a subsequent call for action by the International Society on Thrombosis and Hemostasis (ISTH) Scientific Standardization Committee (SSC) on Fibrinolysis, ${ }^{10}$ we thought it worthwhile to investigate current test practice from participants of the Royal College of Pathologists of Australasia Quality Assurance Programs (RCPAQAP). For this purpose, we constructed a simple survey using Survey Monkey that we asked participants to complete voluntarily. The number of Ddimer results being reported to RCPAQAP in 2020 is 515, representing a total of 407 participants (note that many participants report D-dimer values on multiple instruments). After exclusion of duplicate entries, 100 participants $(24.6 \%$ of active participants) provided answers to the survey questions. Although this is arguably a minor sampling of the total possible pool, it is not unusual for a voluntary survey, and still provides a reasonable snapshot of current status and associated problems. Results are summarised in Fig. 1. The breakdown of participant laboratory types (as self determined by participants) is shown in Fig. 1A, and indicates respondents as mostly deriving from publicly funded facilities. The breakdown of manufacturer reagents in use by participants (Fig. 1B) indicates two main reagents in use by almost $80 \%$ of participants; however, in total, seven different reagents are currently in use. Most participants report in FEU units (Fig. 1C), which also tends to be that recommended by most manufacturers. ${ }^{9}$ Worryingly, $8 \%$ of respondents reported that they did not know whether they reported in FEU or DDU. Most laboratories reported in $\mathrm{mg} / \mathrm{L}$ (Fig. 1D), which is also the current recommendation from the RCPA. ${ }^{11}$ However, four other units were alternatively reported, with $\mathrm{ng} / \mathrm{mL}$ and $\mu \mathrm{g} / \mathrm{mL}$ each being reported by $7-20 \%$ of participants. Most participants reported use of a normal cut-off value of ' 0.5 ' as representing the 'cross over line' for abnormal D-dimer values (Fig. 1E). Naturally, these sites were also predominantly reporting in FEU and $\mathrm{mg} / \mathrm{mL}$. However, a substantial number of laboratories used either ' 500 ' or ' 0.25 ' as the cut-off value. Moreover, 15 (15\%) participants reported 'other' different and quite varied cut- 